\title{
microRNA-133 inhibits cell proliferation, migration and invasion in prostate cancer cells by targeting the epidermal growth factor receptor
}

\author{
$\mathrm{JUN} \mathrm{TAO}^{1 *}$, DEYAO WU ${ }^{2 *}, \mathrm{BIN} \mathrm{XU}^{3}$, WEICHUN QIAN ${ }^{4}$, \\ PENGCHAO LI ${ }^{1}$, QIANG LU ${ }^{1}$, CHANGJUN YIN ${ }^{1}$ and WEI ZHANG ${ }^{1}$ \\ ${ }^{1}$ Department of Urology, First Affiliated Hospital of Nanjing Medical University, Nanjing 210029; \\ ${ }^{2}$ Department of Urology, The Fourth Affiliated Hospital of Nantong Medical College, Yancheng City No. 1 People's Hospital, \\ Nanjing; ${ }^{3}$ Department of Urology, Affiliated Zhongda Hospital of Southeast University, Nanjing 210009; \\ ${ }^{4}$ Department of Cardiology, Nanjing First Hospital, Nanjing Medical University, Nanjing 210006, P.R. China
}

Received December 16, 2011; Accepted February 17, 2012

DOI: $10.3892 / o r .2012 .1711$

\begin{abstract}
It has been shown that regulation of EGFR expression in prostate cancer cells is mostly at the transcriptional level. microRNA-133 (miR-133) has long been recognized as a muscle-specific miRNA which may regulate myoblast differentiation and participate in many myogenic diseases. Recently, it has been reported that miR-133 is also involved in other tumors, such as bladder cancer, esophageal cancer and may regulate cell motility in these cancer cells. In the present study, we examined the expression and effects of miR-133 in two hormone-insensitive prostate cancer cell lines. The expression of miR-133a and miR-133b were analyzed by quantitative RT-PCR. After transfection of miR-133a and miR-133b, cell viability assay, luciferase assay, western blot analysis, cell migration and invasion assay were conducted in DU145 and PC3 cells. In this study, we showed that miR-133a and miR-133b are expressed at the detection limit in two hormoneinsensitive prostate cancer cell lines, PC3 and DU145. Ectopic expression of miR-133 inhibited cell proliferation, migration and invasion in these cells. We also provide the first evidence that miR-133 may target EGFR. Our study provided the first glimpse of the functional role of miR-133 in two hormoneindependent prostate cancer cell lines. These results may add to our knowledge on the molecular basis of prostate cancer progression.
\end{abstract}

Correspondence to: Professor Qiang Lu, Department of Urology, First Affiliated Hospital of Nanjing Medical University, 300 Guangzhou Road, Nanjing 210029, P.R. China

E-mail: luqiang0405@163.com

${ }^{*}$ Contributed equally

Key words: miR-133, epidermal growth factor receptor, prostate cancer

\section{Introduction}

Prostate cancer is the most common malignancy and the second most common cause of cancer death in American men with 192,280 newly diagnosed cases and 27,360 disease related deaths in 2009 (1). Indeed, an obvious stage shift was witnessed during recent two decades after the wide use of PSA testing. Currently, more and more prostate cancers are diagnosed at early stage and effectively treated with local therapies (2). However, since prostate cancer is a heterogeneous entity, there are still advanced diseases at diagnosis or after initial therapies, and this subgroup of the disease accounts for the majority of disease specific mortality. Thus, understanding the molecular basis of more advanced disease and developing novel treatments are critical to further improve the survival of prostate cancer patients.

Androgen deprivation therapy (ADT) is the major armament for treating advanced prostate cancer and all prostate cancers initially respond to ADT. However, after the initial period, most prostate cancers will evolve into castration refractory (CR) diseases (3). CR prostate cancers are associated with more malignant and aggressive phenotypes. The exact mechanisms underlying the development of CR are still undetermined. There are several tangled routes contributing to the androgen-independent growth of prostate cancers, among which epidermal growth factor (EGF) and its receptor (EGFR)mediated pathway has assumed considerable importance due to its overactivation in prostate cancers and cross-talk with the androgen pathway (4). EGFR overexpression has been linked to progression from androgen-responsive disease to CR phenotypes and EGFR signaling could activate androgen receptor pathway even in the circumstances of androgen deprivation. Furthermore, EGFR itself may be under the regulation of androgen signaling pathway. This regulation seems to be negative in normal prostate cells but positive in prostate cancer cells, especially in CR cancer cells (5).

It has been shown that regulation of EGFR expression in prostate cancer cells is mostly at transcriptional level (6). However, gene expression regulation is a complex event 
Table I. Sequence of miR-18a mimics, miR-18a antisense oligonucleotides (ASO), their controls and DICER siRNA.

\begin{tabular}{|c|c|}
\hline Name & Description \\
\hline miR-18a mimics & Double-strand mimics to mature miR-18a \\
\hline Forward & 5'-UAAGGUGCAUCUAGUGCAGAUAG-3' \\
\hline Reverse & 5'-AUCUGCACUAGAUGCACCUUAUU-3' \\
\hline miR-18a inhibitor & $\begin{array}{l}\text { Single-strand antisense oligonucleotides with 2'-O methylation } \\
\text { 5'-CUAUCUGCACUAGAUGCACCUUA-3' }\end{array}$ \\
\hline DICER siRNA 1 & siRNA designed for targeting DICER mRNA (position at 1897) \\
\hline Forward & 5'-GGGCACCCAUCUCUAAUUATT-3' \\
\hline Reverse & 5'-UAAUUAGAGAUGGGUGCCCTT-3' \\
\hline DICER siRNA 2 & siRNA designed for targeting DICER mRNA (position at 2123) \\
\hline Forward & 5'-GGCCAUUGGACACAUCAAUTT-3' \\
\hline Reverse & 5'-AUUGAUGUGUCCAAUGGCCTT-3' \\
\hline DICER siRNA 3 & siRNA designed for targeting DICER mRNA (position at 4343) \\
\hline Forward & 5'-GGUGGUGUCAAUAUUUGAUTT-3' \\
\hline Reverse & 5'-AUCAAAUAUUGACACCACCTT-3' \\
\hline NC_mimics & Negative control for mimics and siRNA' \\
\hline Forward & 5'-UUCUCCGAACGUGUCACGUTT-3' \\
\hline Reverse & 5'-ACGUGACACGUUCGGAGAATT-3' \\
\hline \multirow[t]{2}{*}{ NC_inhibitor } & Negative control for antisense oligonucleotides \\
\hline & 5'-CAGUACUUUUGUGUAGUACAA-3' \\
\hline
\end{tabular}

in living cells including genomic or epigenomic switch, transcriptional control, and post-transcriptional regulation. Therefore, it is still possible that EGFR might be regulated after its transcription in prostate cancer cells. In addition to genetic factors, microRNAs (miRNAs) are emerging as small regulators finely modulating gene expression post-transcriptionally by binding to mRNA 3 ' untranslated region (3'UTR) (7). miRNAs have been revealed to be involved in almost every cellular activity including proliferation, differentiation, apoptosis, maintenance of stemness and carcinogenesis. In prostate cancers there have been many reports showing that dysregulated miRNAs might be involved in the development and progression of this disease (4,7-9). However, whether miRNAs take part in the transition from ADT sensitive status to insensitiveness is still not known mainly due to the lack of clinical samples for profiling study. microRNA-133 (miR-133) has long been recognized as a muscle specific miRNA which may regulate myoblast differentiation and participate in many myogenic diseases (10-12). Recently, it has been reported that miR-133 was also involved in other tumors, such as bladder cancer, esophagus cancer and might regulate cell motility in these cancer cells (13-15). In a profiling study, miR-133a was found downregulated in prostate cancer tissues compared with non-cancer tissues (9). However, other profiling studies did not reveal any significance of miR-133 dysregulation.

In this study, we showed that miR-133a and miR-133b were expressed at the detection limit in the hormone insensitive prostate cancer cell lines PC3 and DU145. Ectopic expression of miR-133 inhibited cell proliferation, migration and invasion in these cells, indicating tumor suppressive role of these miRNAs in prostate cancer cells, at least in androgen independent cell lines. We also provide the first evidence that miR-133 might target EGFR, which might link the microRNA and the mechanisms of hormone insensitivity.

\section{Materials and methods}

Cell lines and culture conditions. Prostate cancer cell lines DU145 and PC3 were purchased from cell bank of Chinese Academy of Science (Shanghai, China). The cells were cultured in RPMI-1640 medium supplemented with $10 \%$ heat-inactivated fetal bovine serum, penicillin $(100 \mathrm{U} / \mathrm{ml})$, and streptomycin $(100 \mathrm{mg} / \mathrm{l})$ at $37^{\circ} \mathrm{C}$ in a humidified atmosphere containing $5 \% \mathrm{CO}_{2}$. Cells were subcultured every 2 days using trypsin/EDTA solution (saline containing $0.05 \%$ trypsin, $0.01 \mathrm{M}$ sodium phosphate and $0.53 \mu \mathrm{M}$ EDTA, $\mathrm{pH}$ 7.4).

Quantitative $R T-P C R$ for $m i R-133$. Total RNA was extracted from tissues using TRIzol reagent (Invitrogen). Real-time qRT-PCR for miR-133a/b was performed with Sybergreen microRNA assay (Shanghai GenePharma Co., Ltd., Shanghai, China) according to the manufacturer's protocol. Briefly, a total $500 \mathrm{ng}$ RNA was used for the initial reverse transcription reaction using gene specific stem-loop RT primer available in the kit. Real-time PCR was performed on AB7300 thermo-recycler (Applied Biosystems) using miR-133 primer set and double-stranded binding dye SYBRGreen. GAPDH was used as internal control. The primers for GAPDH were: F-GAAATCCCATCACCATCTTCCAGG, R-GAGCCCCAGCCTTCTCCATG. Every sample was replicated three times with no RT and no template control. Data were analyzed by comparing $\mathrm{Ct}$ values. 
Table II. Primers used for clone EGFR mRNA 3'UTR.

\begin{tabular}{ll}
\hline Name & \multicolumn{1}{c}{ Description } \\
\hline $\mathrm{S} 1+\mathrm{S} 2$ & Fragment containing both binding sites \\
Forward & CCCAAGCTTTTAGGGCTCATACTATCCTCCGTGG \\
Reverse & CGAGCTCAGCTGCTCTGAAATCTCCTTTAGCC \\
$\mathrm{S} 1$ only & Fragment containing only S1 site \\
Forward & CCCAAGCTT TTAGGGCTCATACTATCCTCCGTGG \\
Reverse & CGAGCTCAATAATAACTCGGATTCCAGCCCAC \\
S2 only & Fragment containing only S2 site \\
Forward & CCCAAGCTTGTCTGTGGGTCTAAGAGCTAATGCG \\
Reverse & CGAGCTCATCTCCTTTAGCCATCACCCCAACC \\
None & Fragment containing no binding site \\
Forward & CCCAAGCTTGTCTGTGGGTCTAAGAGCTAATGCG \\
Reverse & CGAGCTCAAACTCAGTATGCTGCCCCTGTCTT \\
\hline
\end{tabular}

Transfection of microRNA mimics and luciferase reporter plasmid. Mature miR-133a mimics, miR-133b mimics, and scrambled control were designed and synthesized by GenePharma Co., Ltd. The sequence of miR-133a mimics was 5'-UUUGGUCCCCUUCAACCAGCUG-3'. The sequence of miR-133b mimics was 5'-UUUGGUCCCCUUCAACCAGC UA-3'. The control was 5'-UUCUCCGAACGUGUCAC GUTT-3'. EGFR 3'UTR fragments were amplified using synthetic primers added to enzymatic cutting sites. The restriction endonuclease used for forward primers was HindIII, and that for reverse primers was SacI. The fragments were then cloned into pMIR-Report system (Ambion, Austin, TX, USA). Table I lists the primer sets used in 3'UTR clone. The insertion fragment was confirmed by DNA sequencing. Cell transfection and cotransfection were performed using Lipofectamine 2000 (Invitrogen) according to the manufacturer's protocol.

Cell growth/cell viability assay. Cell proliferation was determined using the MTT method. Cells transfected with miR-133a/b mimics or controls were seeded in 96-well plates at a density of 3,000 cells/well. Cell viability was documented every $24 \mathrm{~h}$ for 5 days following the manufacturer's protocol. Briefly, $20 \mu 1$ 3-(4,5-dimethyl-2-thiazoyl)-2,5-diphenyl2H-tetrazolium bromide (MTT) solution was added into each well and incubated at $37^{\circ} \mathrm{C}$ for $4 \mathrm{~h}$. The plates were briefly centrifuged, and the purple colored precipitates of formazan were dissolved in $200 \mu \mathrm{l}$ DMSO. Absorbance was measured at $490 \mathrm{~nm}$ in an ELISA reader (Bio-Rad Laboratories, Richmond, CA, USA). Six-well replication was used for all time points in each group. Proliferation curves were drawn on the basis of mean absorbance at each time point.

Cell migration and invasion assay. The transfected cells (miR-133a mimics, miR-133b mimics and scrambled control) growing in the log phase were treated with trypsin and re-suspended as single-cell solutions. A total of $1 \times 10^{5}$ cells in $0.2 \mathrm{ml}$ of serum-free RPMI-1640 medium were seeded on an $8 \mu \mathrm{m}$-pore polycarbonate membrane Boyden chamber insert in a transwell apparatus (Costar, Cambridge, MA), either coated with or without Matrigel (BD Biosciences, San Jose, CA). RPMI-1640 $(600 \mu \mathrm{l})$ containing 20\% FBS was added to the lower chamber. After the cells were incubated for $12-24 \mathrm{~h}$ at $37^{\circ} \mathrm{C}$ in a $5 \% \mathrm{CO}_{2}$ incubator, cells on the top surface of the insert were removed by wiping with a cotton swab. Cells that migrated to the bottom surface of the insert were fixed in $100 \%$ methanol for $2 \mathrm{~min}$, stained in $0.5 \%$ crystal violet for $2 \mathrm{~min}$, rinsed in PBS and then subjected to microscopic inspection (original magnification, $\mathrm{x} 200$ ). Values for invasion and migration were obtained by counting five fields per membrane and represent the average of three independent experiments.

Luciferase assay. Cells were plated in a 12-well plate at $\sim 90 \%$ confluence and cotransfected with $0.5 \mu \mathrm{g}$ reporter

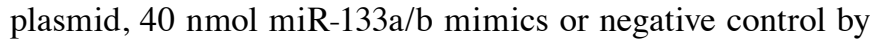
Lipofectamine 2000. Each sample was also cotransfected with $0.05 \mu \mathrm{g}$ pRL-CMV plasmid expressing Renilla Luciferase (Promega) as an internal control for transfection efficiency. Luciferase assay was performed $48 \mathrm{~h}$ after transfection using Dual Luciferase Reporter Assay System (Promega). Firefly luciferase activity was normalized to Renilla luciferase activity for each transfected well. Each assay was replicated 3 times. The primers used for clone EGFR mRNA 3'UTR are given in Table II.

Western blot analysis. Primary antibodies used in this study including EGFR, ERK, phosphorylated ERK, AKT, phosphorylated AKT and MMP-2 were products of Bioworld Technology, Inc. (Louis Park, MN, USA). Antibodies against $\beta$-actin were purchased from Santa Cruz Biotechnology. Total protein of cells were prepared using RIPA lysis buffer. Protein concentration in the resulting lysate was determined using the bicinchoninic acid assay. Equal amounts of protein were loaded on an SDS-PAGE and transferred to PVDF membrane. After blocking with 5\% degreased milk in TBST, the membranes were incubated overnight with appropriately diluted primary antibody at $4^{\circ} \mathrm{C}$. Next, they were washed and incubated with the 
A

\section{DU145}

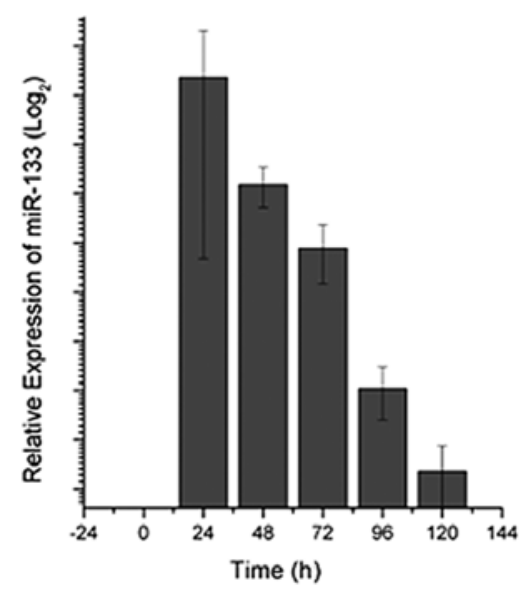

B

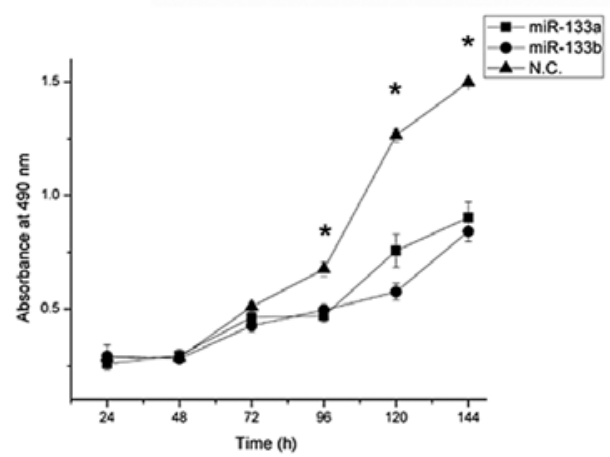

PC3

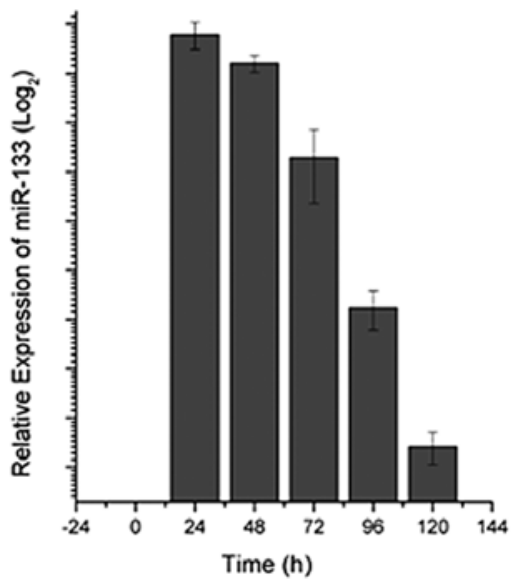

PC3

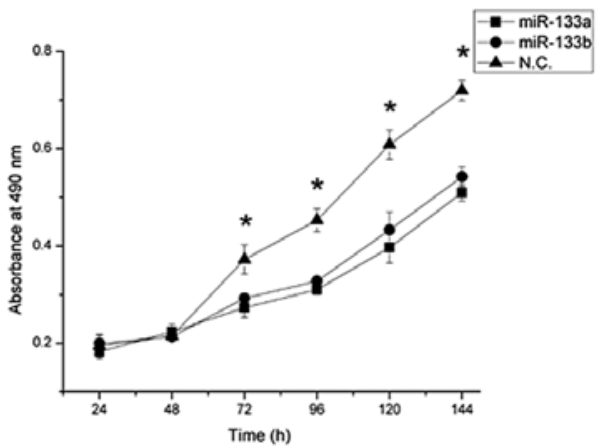

Figure 1. (A) The expression of miR-133a/b in DU145 and PC3 cells. The basal expression of miR-133a/b in DU145 and PC3 cells were only at detectable limitation. The expression of miR-133 was dramatically increased until $120 \mathrm{~h}$ after transfection of miR-133. (B) Viability of DU145 and PC3 cells after transfection of miR-133a/b. Cell viability was analyzed using an MTT assay. The results indicated that upregulation of miR-133a and miR-133b significantly suppressed cell proliferation.

corresponding horseradish peroxidase conjugated secondary antibody at 1:2000 dilution in TBST. The blot was developed with ECL solution (Pierce) and photographed by FluorChem Imaging System (Alpha Innotech). The intensity of each spot was read and analyzed with AlphaEaseFC software.

Statistical analysis. Data are presented as the mean \pm SD, and compared using Student's t-test in Stata 10.0 (College Station, TX, USA). Double-tailed P-value $<0.05$ was considered to be statistically significant.

\section{Results}

Ectopic expression of miR-133a/b suppresses cell proliferation in prostate cancer cell lines. Firstly, we surveyed the basal expression of miR-133a/b in DU145 and PC3 cells as well as its expression after transfection of miR-133a every $24 \mathrm{~h}$. miR-133a and miR-133b were indiscernible due to only one base difference between them. As expected, the basal expression of miR-133a/b was only at detection limit, which was too low to be seen in Fig. 1A. After transfection of miR133a, the expression level was dramatically increased for about $120 \mathrm{~h}$. To investigate the influence of miR-133a/b on cell proliferation, we performed an MTT assay. As shown in Fig. 1B, upregulation of miR-133a and miR-133b significantly suppressed cell proliferation. It was revealed that after $144 \mathrm{~h}$ of treatment, the inhibition rate of miR-133a reached $39.34 \pm 4.6 \%$ in DU145 cells and $29.20 \pm 3.8 \%$ in PC3 cells and that of miR-133b reached $43.80 \pm 3.3 \%$ in DU145 cells and $24.65 \pm 4.3 \%$ in PC3 cells. These results indicate that $\mathrm{miR}-133 \mathrm{a} / \mathrm{b}$ may play a suppressive role in prostate cancer cells in vitro.

miR-133a/b inhibits cell migration and invasion in vitro. To measure the effect of miR-133a/b on tumor cell migration, transfected cells growing in the log phase were collected and cultured on transwell apparatus. After $12 \mathrm{~h}$ incubation, cell migration was significantly decreased in miR-133a and miR-133b groups than in control group $(\mathrm{P}<0.05)$ (Fig. 2A). Using transwell apparatus pre-coated with Matrigel, we examined the effects of miR-133a/b on cell invasiveness. After $24 \mathrm{~h}$ incubation, $\mathrm{miR}-133 \mathrm{a} / \mathrm{b}$ transfected cells showed significantly decreased invasiveness compared with the control cells $(\mathrm{P}<0.05)$ (Fig. 2B). These results indicate that miR-133a and miR-133b inhibit the cell migration and invasion in prostate cancer cell lines. 
A

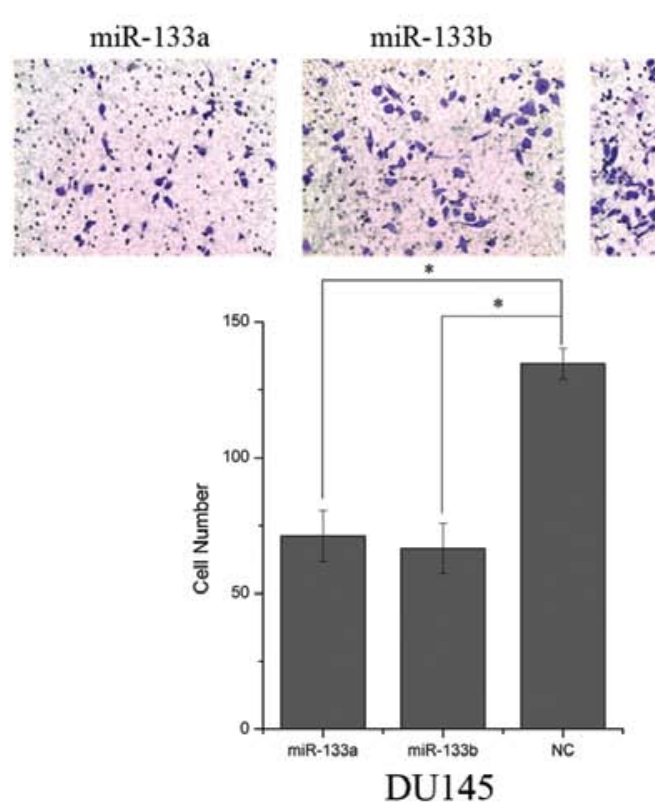

$\mathrm{NC}$

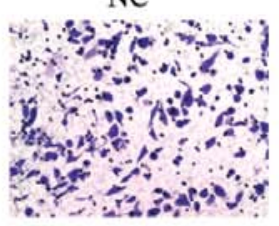

ano
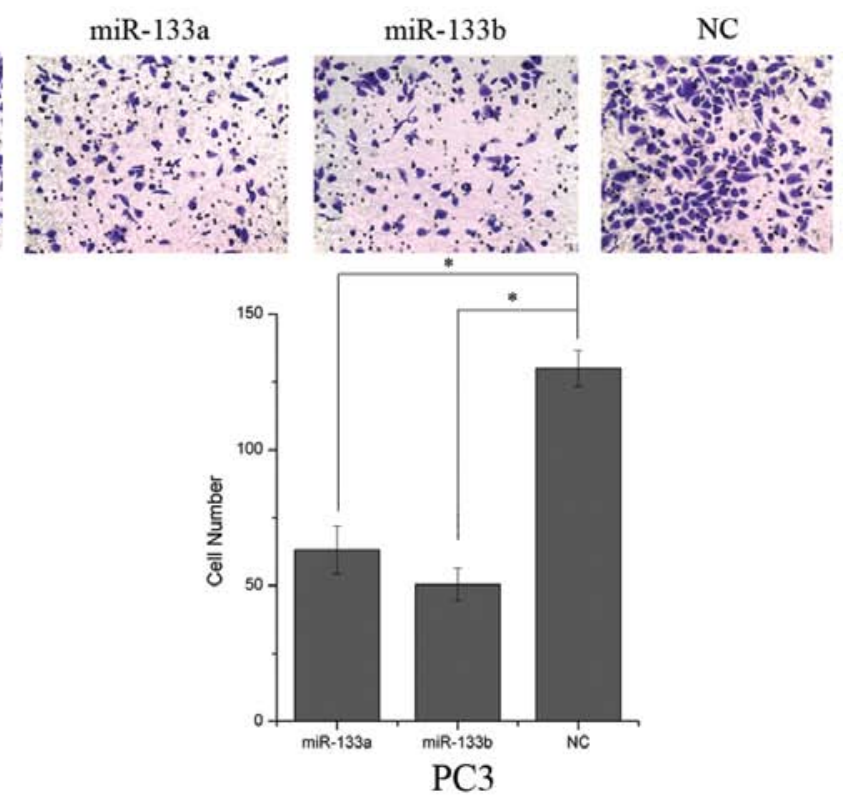

B
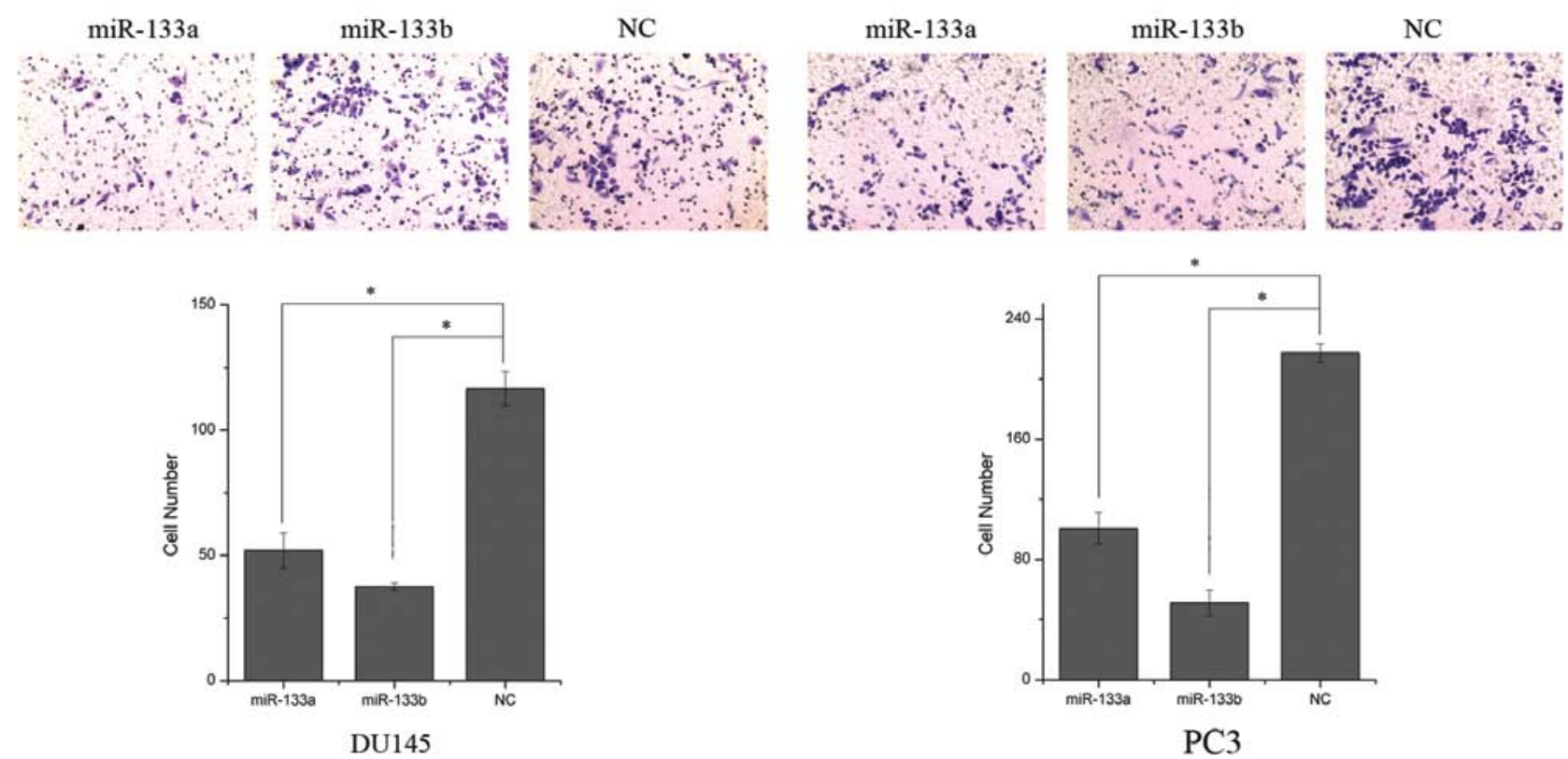

Figure 2. miR-133a/b inhibits cell migration and invasion. (A) Representative photographs and statistic plots of the migration assay in DU145 and PC3 cells. After 12-h incubation, the number of DU145 and PC 3 cells that transversed the transwell membrane was decreased after transfection of miR-133a/b (Student's t-test, $\mathrm{P}<0.05$ ). (B) Representative photographs and statistic plots of the invasion assay in DU145 and PC3 cells. After 24-h incubation, the number of DU145 and PC 3 cells that transversed the transwell membrane pre-coated with Matrigel was decreased after transfection of miR-133a/b (Student's t-test, ${ }^{*} \mathrm{P}<0.05$ ).

EGFR is downregulated after overexpression of miR-133a/b, which in turn inactivates its downstream effectors. In bioinformatic studies, we identified EGFR as a putative target of miR-133a/b. We noted that EGFR played an important role in hormone-independent prostate cancer cell lines including DU145 and PC3. We performed western blot analysis to explore whether EGFR protein level was decreased after ectopic overexpression of miR-133a/b. As shown in Fig. 3A, EGFR was significantly lowered in both DU145 and PC3 cell lines $72 \mathrm{~h}$ after transfection of miR-133a/b, respectively. Furthermore, downstream signaling pathways of EGFR were also inactivated as phosphorylated ERK and AKT were both lessened after miR-133a/b treatment (Fig. 3B). MMP-2, as an effector of EGFR pathway and mediating cell migration and invasion, was also downregulated at the same time point after transfection of miR-133a/b (Fig. 3C). Thus, miR-133a/b reduces the protein level of EGFR in prostate cancer cells whereby inactivating the downstream signals.

EGFR is a direct target of miR-133a/b. According to a computational prediction, there were two binding sites (S1 and S2) of miR-133a/b on EGFR 3'UTR, as illustrated in Fig. 4A. The conserved binding site is $28 \mathrm{bp}$ from the coding region, while $\mathrm{S} 2$ is less conserved and $856 \mathrm{bp}$ from the coding region. 
A DU145
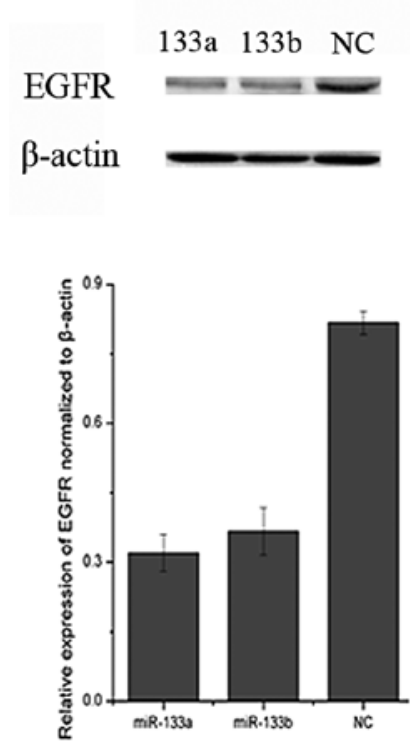

C
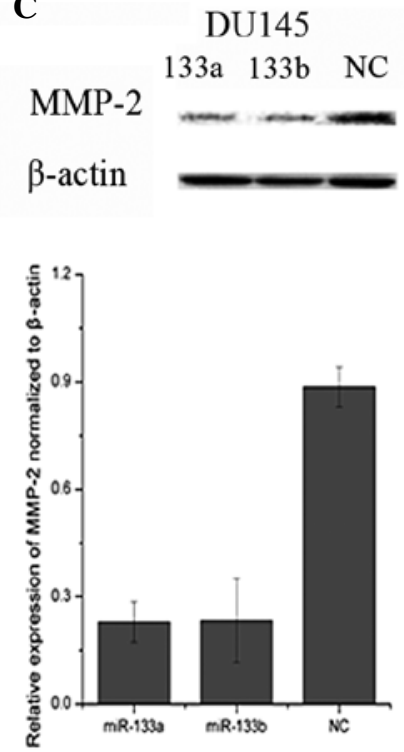

PC3
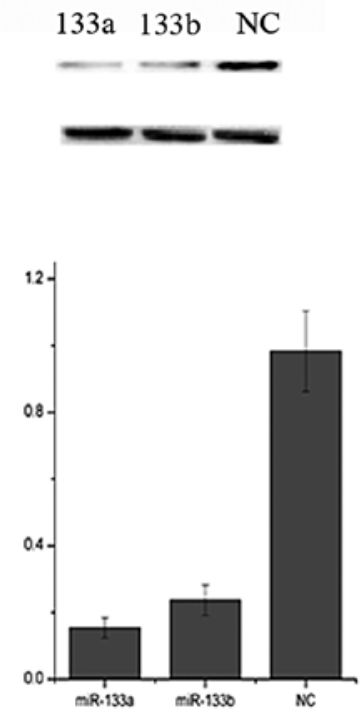

PC3

133a 133b NC
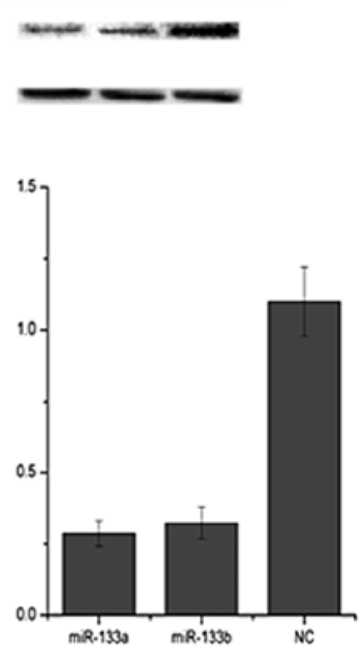

B

DU145

PC3

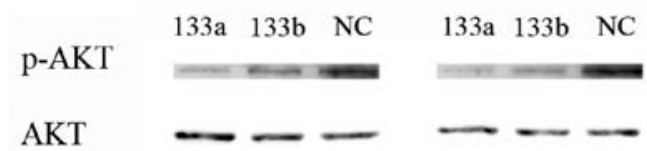

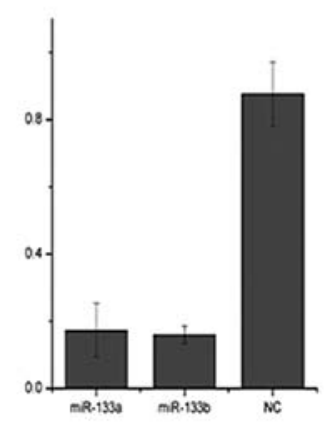

p-ERK

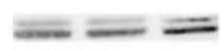

ERK
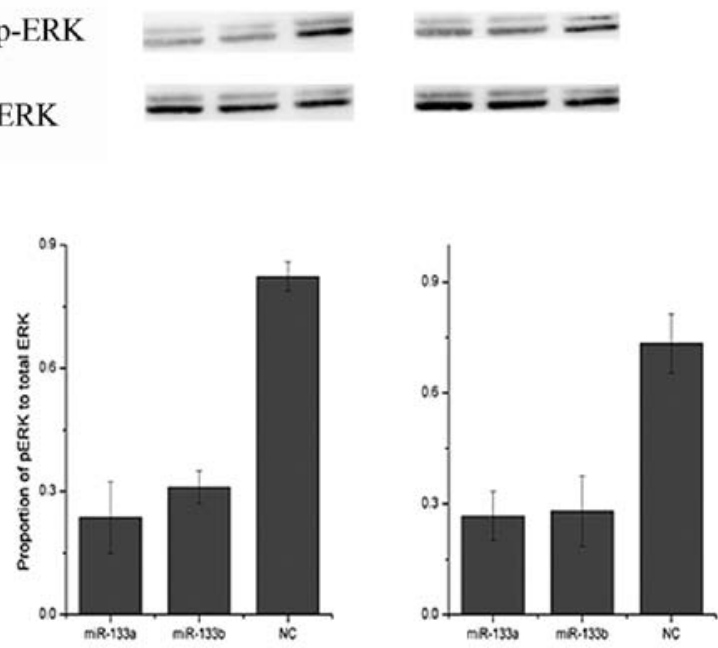

Figure 3. The expression of EGFR, phosphorylated ERK, phosphorylated AKT and MMP-2 in DU145 and PC3 cells $72 \mathrm{~h}$ after transfection of miR-133a/b. (A) EGFR was significantly reduced in both DU145 and PC3 cells after transfection of miR-133a/b. (B) Phosphorylated ERK and AKT were also reduced in DU145 and PC 3 cells after transfection of miR-133a/b. (C) MMP-2 was downregulated at the same time point after transfection of miR-133a/b.

Above all, EGFR may be the direct target of miR-133a/b in vitro.

We then cloned 3 UTR fragments into pMIR-Report vecti Luciferase reporter assays were performed to evaluate whether these sites could directly mediate the expression inhibition. As shown in Fig. 4B, in DU145 cell line, when S1 and S2 sites were both intact, miR-133a induced $85 \%$ suppression in luciferase activity while miR-133b induced $50 \%$ suppression. S1 site alone mediated $82 \%$ inhibition when co-transfected with miR-133a while $40 \%$ inhibition with miR-133b. The S2 site alone only mediated $33 \%$ and $35 \%$ inhibition of lucifrase activity when co-transfected with miR-133a and miR-133b, respectively. Similar results were observed when treated with PC3 cell line. It seemed that miR-133a and miR-133b were slightly different in their affinity to their targets. Moreover, the $\mathrm{S} 1$ site exhibited much more affinity to miR-133a/b than $\mathrm{S} 2$ site while these two sites displayed some synergic effect.

\section{Discussion}

EGFR has long been recognized as a key growth factor mediating hormone sensitivity transition in prostate cancer (16). In this study, we provided concrete evidence that miR-133a/b could suppress the EGFR expression by binding to its 3'UTR in prostate cancer cell line DU145 and PC3, inhibiting cell proliferation, migration as well as invasion. These results elicit the possibility that miR-133 may participate in the regulation of EGFR in castration refractory prostate cancers.

miR-133 is a microRNA family containing miR-133a and miR-133b. miR-133a itself is a multicopy gene with two copies 
A

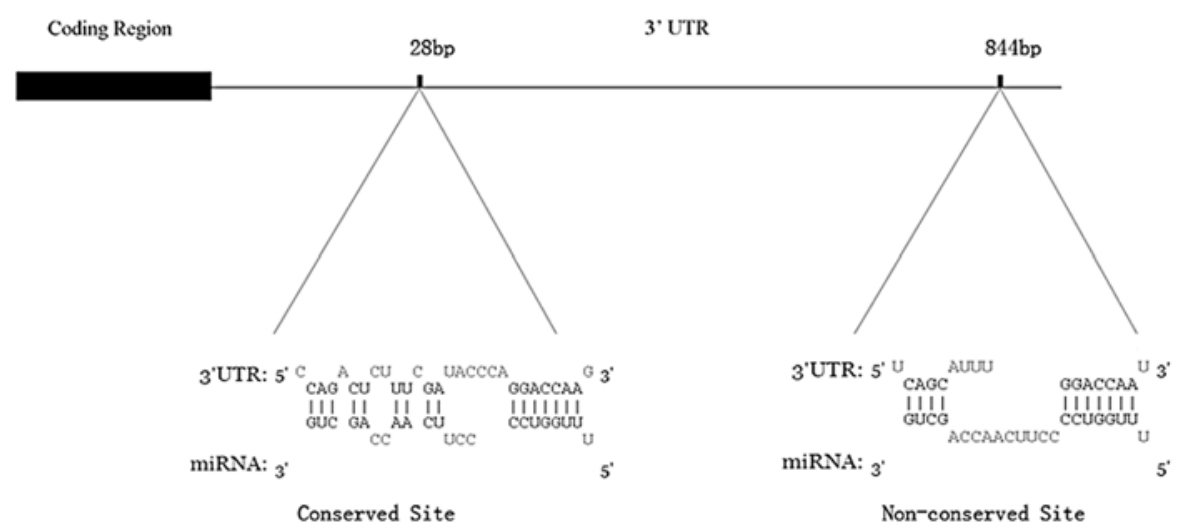

B

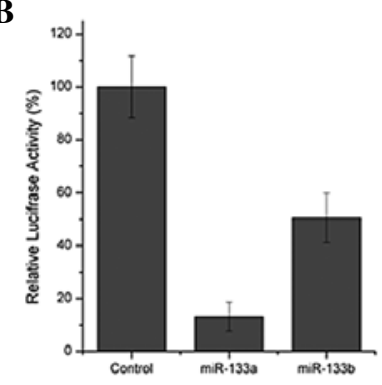

\section{$\mathrm{S} 1+\mathrm{S} 2$}

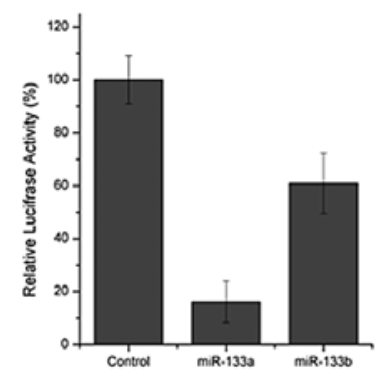
2
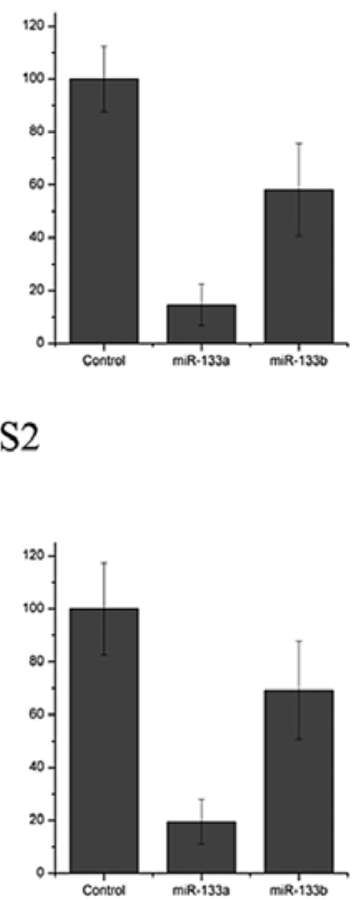

S1
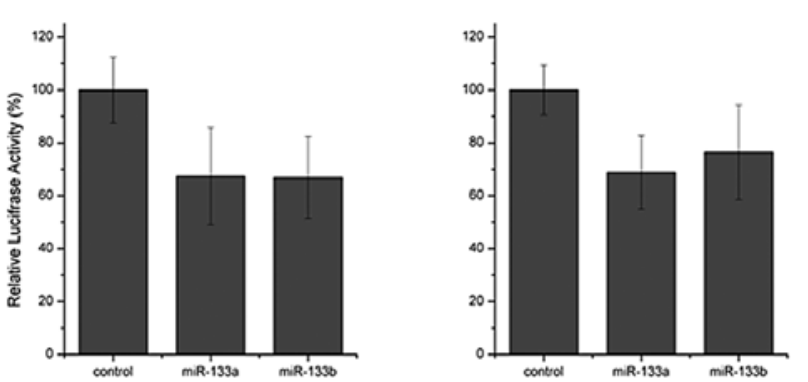

S2
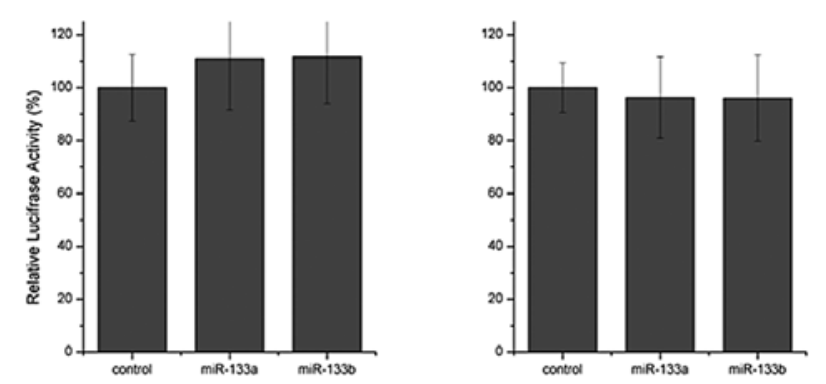

Blank UTR

Figure 4. EGFR is a direct target of miR-18a. (A) Scheme of putative miR-133a/b binding sites on EGFR mRNA 3'UTR. Site 1 (S1) is a highly conserved binding site while site 2 (S2) is slightly conserved. (B) Luciferase reporter assay verified direct binding of miR-133a/b with predicted sites. Luciferase activity was significantly lower when co-transfected with miR-133a/b and vectors containing both S1 and S2 sites in DU145 cells and PC 3 cells. Significant decrease of relative Luciferase activity was also observed when miR-133a/b and vectors containing S1 site were co-transfected. S2 site only rendered a mild but statistically significant effect. Co-transfection with miR-133a/b and blank vector or co-transfection with negative control and vectors containing binding sites were used as controls. Three independent experiments were performed and data are presented as the mean \pm SD.

distributed in chromosome 18 and chromosome 20, both are neighbors to another muscle enriched microRNA miR-1, while miR-133b is located in chromosome 6 and slightly different in base sequence from that of miR-133a, which may manifest nuances in target affinity as shown in this study. Previously, miR-133 has been recognized as a muscle and cardiac specific microRNA which may regulate myogenesis and differentiation. Recently, these miRNAs were revealed to be downregulated in many human malignancies including urinary bladder cancer and prostate cancer. One of the miRNAs in this family, miR-133a, has been shown to inhibit cell proliferation and induce apoptosis by directly binding to a potent oncogene pyruvate kinase type M2 (17). Yoshino et al reported that miR-1 and miR-133a played a tumor suppressive role in bladder cancer by directly targeting transgelin 2 (13). In a recent profiling study, Ambs et al showed that miR-1 and miR-133a were downregulated in prostate cancer tissues and identified several targets of miR-1 in prostate cancer cells (9). However, few studies have focused on miR-133 family including miR-133a and miR-133b.

We revealed that miR-133 was only at the detectable limit in two hormone-independent prostate cancer cell lines, but miR133a and miR-133b could not be discriminated by quantitative RT-PCR method used in this study due to the only one base 
difference at their 3 ' end. Ectopic expression of miR-133a/b significantly suppressed cell proliferation, migration and invasion. These results provided the first evidence that miR-133 might play a tumor suppressive role in prostate cancer cells especially the hormone-independent ones. However, whether these miRNAs truly take part in the development and progression of prostate cancer needs further verification. Among several microRNA profiling studies concerning prostate cancer, only one study showed that miR-133a was downregulated in prostate cancer tissues (9). These divergent results could be attributed to the discrepancy of sampling rather than the insignificance of miR-133. Based on our results miR-133 might be involved in the development of castration refractory diseases, but clinical specimens for this stage of disease are very rare. Further verifications are urgently needed when samples are available.

Our results revealed multiple inhibition effects of miR-133 in DU145 and PC3 cell lines including growth arrest, migration and invasion suppression. In order to identify the target genes accounting for these comprehensive effects, we searched bioinformatic prediction database and noticed EGFR, which might have great importance in the development of androgen-independent prostate cancers and account for the multiple functional alteration. As we showed in this study, there are two binding sites on EGFR mRNA 3'UTR and both can mediate inhibition effect of miR-133. The more conserved site appeared to have greater efficacy than the less conserved one. Additionally, miR133a and miR-133b displayed varied affinity to these sites, which may be attributed to the slight difference in their sequences.

EGFR regulates cell growth, differentiation, motility, adhesion and invasion through interaction with its ligand EGF. EGFR belongs to ErbB oncogene family, which also includes ErbB-2, 3 and 4 and is comprehensively expressed in epithelial cells including prostate cells. Signal transduction after binding to EGF is mainly through two major pathways, one is the MAP kinase pathway and the other is the AKT pathway (18). Overexpression of EGFR and its downstream pathways, including ras-MEK-ERK and PI3K-AKT, are linked to advanced prostate cancer, especially hormoneindependent disease (19). It has been reported that EGFR was highly expressed in DU145 and PC3 cell lines and these cell lines were responsive to EGF stimulation (20). Furthermore, signaling through MAPK pathway may in turn promote phosphorylation of EGFR itself and accelerate the turnover of this receptor. On the other hand, PI3K-AKT pathway may contribute to cell growth, cell motility mainly through its downstream effectors, such as S6 kinase and GSK $3 \beta$. S6 kinase may activate ribosome and contribute to protein synthesis and cell growth while GSK3 $\beta$ may further regulate cell motility and epithelial-mesenchymal transition through snail-E-cadherin pathway (21). In our study, miR133a/b downregulated EGFR, which further inactivated its downstream signaling including MAPK and AKT pathway. As a result, MMP-2, which may be regulated through the GSK3 $\beta$-snail-E-cadherin pathway, was also downregulated after $\mathrm{miR}-133 \mathrm{a} / \mathrm{b}$ transfection.

Our study demonstrated that miR-133a/b may target EGFR in prostate cancer cells and downregulation of
EGFR by ectopic expression of $\mathrm{miR}-133 \mathrm{a} / \mathrm{b}$, this could well explain the functional change observed in these cells, however, there are still several questions unanswered. We still cannot make clear the significance of miR-133 in prostate cancer progression, which may need verification in clinical samples and functional study in other hormone sensitive cell lines. Clearly EGFR is not the sole target of miR-133 in living cells. Further study is needed to fully explain the mechanisms underlying the suppressive effect of miR-133 in prostate cancer cells.

Our study provided the first glimpse of the miR-133's functional role in two hormone independent prostate cancer cell lines. These results may add to our knowledge on the molecular basis of progression of prostate cancer.

\section{Acknowledgements}

This study was supported by the program of the Key Medical Department of the Jiangsu Province-Department of General Surgery of the Jiangsu Province Hospital and the Department of Urology of the Jiangsu Province Hospital.

\section{References}

1. Jemal A, Siegel R, Ward E, Hao Y, Xu J and Thun MJ: Cancer statistics, 2009. CA Cancer J Clin 59: 225-249, 2009.

2. Pashayan N,Pharoah P,Neal DE, et al: Stage shift in PSA-detected prostate cancers - effect modification by Gleason score. J Med Screen 16: 98-101, 2009.

3. Mottet N, Bellmunt J, Bolla M, et al: EAU guidelines on prostate cancer. Part II: Treatment of advanced, relapsing, and castration-resistant prostate cancer. Eur Urol 59: 572-583, 2011.

4. Wang L, Tang H, Thayanithy V, et al: Gene networks and microRNAs implicated in aggressive prostate cancer. Cancer Res 69: 9490-9497, 2009.

5. Traish AM and Morgentaler A: Epidermal growth factor receptor expression escapes androgen regulation in prostate cancer: a potential molecular switch for tumour growth. Br J Cancer 101: 1949-1956, 2009.

6. Cohen MB and Rokhlin OW: Mechanisms of prostate cancer cell survival after inhibition of AR expression. J Cell Biochem 106: 363-371, 2009.

7. Porkka KP, Pfeiffer MJ, Waltering KK, Vessella RL, Tammela TL and Visakorpi T: microRNA expression profiling in prostate cancer. Cancer Res 67: 6130-6135, 2007.

8. Tong AW, Fulgham P, Jay C, et al: microRNA profile analysis of human prostate cancers. Cancer Gene Ther 16: 206-216, 2009.

9. Ambs S, Prueitt RL, Yi M, et al: Genomic profiling of microRNA and messenger RNA reveals deregulated microRNA expression in prostate cancer. Cancer Res 68: 6162-6170, 2008.

10. Rao PK, Missiaglia E, Shields L, et al: Distinct roles for miR-1 and miR-133a in the proliferation and differentiation of rhabdomyosarcoma cells. FASEB J 24: 3427-3437, 2010.

11. Rao PK, Kumar RM, Farkhondeh M, Baskerville $S$ and Lodish HF: Myogenic factors that regulate expression of musclespecific microRNAs. Proc Natl Acad Sci USA 103: 8721-8726, 2006.

12. Bostjancic E, Zidar N, Stajer D and Glavac D: microRNAs miR-1, miR-133a, miR-133b and miR-208 are dysregulated in human myocardial infarction. Cardiology 115: 163-169, 2010.

13. Yoshino H, Chiyomaru T, Enokida H, et al: The tumoursuppressive function of miR-1 and miR-133a targeting TAGLN2 in bladder cancer. Br J Cancer 104: 808-818, 2011.

14. Uchida Y, Chiyomaru T, Enokida H, et al: miR-133a induces apoptosis through direct regulation of GSTP1 in bladder cancer cell lines. Urol Oncol: Mar 9, 2011 (Epub ahead of print).

15. Kano M, Seki N, Kikkawa N, et al: miR-145, miR-133a and miR-133b: tumor suppressive miRNAs target FSCN1 in esophageal squamous cell carcinoma. Int J Cancer 127: 2804-2814, 2010. 
16. Neto AS, Tobias-Machado M, Wroclawski ML, Fonseca FL Pompeo AC and Del Giglio A: Molecular oncogenesis of prostate adenocarcinoma: role of the human epidermal growth factor receptor 2 (HER-2/neu). Tumori 96: 645-649, 2010.

17. Wong TS, Liu XB, Chung-Wai Ho A, Po-Wing Yuen A, Wai-Man Ng R and Ignace Wei W: Identification of pyruvate kinase type M2 as potential oncoprotein in squamous cell carcinoma of tongue through microRNA profiling. Int J Cancer 123: 251-257, 2008 .

18. Kim HG, Kassis J, Souto JC, Turner T and Wells A: EGF receptor signaling in prostate morphogenesis and tumorigenesis. Histol Histopathol 14: 1175-1182, 1999.
19. Hernes E, Fossa SD, Berner A, Otnes B and Nesland JM: Expression of the epidermal growth factor receptor family in prostate carcinoma before and during androgen-independence. Br J Cancer 90: 449-454, 2004

20. Kharait S, Dhir R, Lauffenburger D and Wells A: Protein kinase Cdelta signaling downstream of the EGF receptor mediates migration and invasiveness of prostate cancer cells. Biochem Biophys Res Commun 343: 848-856, 2006.

21. Gan Y, Shi C, Inge L, Hibner M, Balducci J and Huang Y: Differential roles of ERK and Akt pathways in regulation of EGFR-mediated signaling and motility in prostate cancer cells. Oncogene 29: 4947-4958, 2010. 